\title{
Long Term Responses with Cetuximab Therapy in Glioblastoma Multiforme
}

\section{Cristóbal Belda-Iniesta ${ }^{1, *}$ \\ Javier de Castro Carpeño ${ }^{1}$ \\ Enrique Casado Saenz ${ }^{1}$ \\ Manuel Gutiérrez ${ }^{2}$ \\ Rosario Perona ${ }^{3}$ \\ Manuel González Barón ${ }^{1}$}

${ }^{1}$ Translational Oncology Unit CSIC-UAM; Medical Oncology Division; Hospital Universitario La Paz; Universidad Autonoma de Madrid; Madrid, Spain

${ }^{2}$ Department of Pathology; Hospital Universitario La Paz; Madrid, Spain

${ }^{3}$ Translational Oncology Unit CSIC-UAM; Instituto de Investigaciones Biomedicas "Alberto Sols", CSIC; Arturo Duperier; Madrid, Spain

*Correspondence to: Cristóbal Belda-Iniesta; Medical Oncology Division; Hospital Universitario La Paz; Paseo de la Castellana 261; Madrid 28046 Spain; Tel.: +34.9.1207.1157; ext. 1138; Fax: +34.9.1727.7118; Email: cbelda@iib.vam.es

Received 05/12/06; Accepted 06/23/06

Previously published online as a Cancer Biology \& Therapy E-publication: http://www.landesbioscience.com/journals/cbt/abstract.php?id $=3118$

\section{KEY WORDS}

glioblastoma multiforme, Cetuximab, EGFR, targeted therapy, third line

\section{ABSTRACT}

Glioblastoma multiforme (GBM) is responsible for most of the deaths associated with primary brain tumors. Standard treatment includes maximal surgical resection followed by chemotherapy and concomitant radiotherapy. Most patients, however, recur shortly after treatment. Second line treatment has little efficacy and the majority of patients die soon from the disease. Recent advances in molecular biology have implicated the epidermal growth factor receptor (EGFR) signaling pathways in the progression and resistance to standard therapies for GBM. This has prompted the evaluation of EGFR tyrosine- kinase inhibitors with encouraging results. Cetuximab is a monoclonal antibody targeted against the extra cellular domain of the EGFR with activity against different tumor types, either alone or in combination with chemotherapy and/or radiation therapy. Here we describe three patients with recurrent, heavily pretreated, EGFR expressing GBM who responded to treatment with single agent cetuximab.

Glioblastoma multiforme (GBM) is the most frequent primary malignant brain tumor in adults. Current treatment includes maximal surgical resection followed by adjuvant treatment with chemotherapy, starting with low dose followed by full dose of temozolomide for six months, combined with radiotherapy. In a phase III trial, this strategy resulted in a median survival of 14.6 months and a $26.5 \%$ two-year survival. However, only $10.7 \%$ of patients remained progression free at two years, reflecting an elevated rate of recurrence ${ }^{1}$ and most patients with disease progression die shortly thereafter. New treatment strategies are therefore urgently needed in this setting.

Recent advances in molecular biology have implicated the EGFR signaling pathways in the progression and resistance to standard therapies in GBM. ${ }^{2}$ Overexpression of this receptor is present in $40 \%$ of patients with GBM and preclinical models have suggested the potential role for EGFR targeted therapies for this condition. ${ }^{3}$ In fact, the EGFR tyrosine-kinase inhibitors gefitinib and erlotinib are being evaluated in ongoing clinical trials in this disease. The impact of this strategy in overall survival remains, however, unclear. ${ }^{4}$ Recent data with EGFR kinase inhibitors suggest a protein profile associated with responsiveness to these drugs. ${ }^{5}$ However, coexpression of EGFRvIII (a pathological variation of EGFR that lacks the extracellular domain) and PTEN are needed to effectively predict clinical and radiological responses in progressive GBM patients. No correlation was observed with wild-type EGFR expression. Probably, disparate results reported with EGFR kinase inhibitors are correlated with the absence of this protein profile. In addition, GBM progression is associated with VEGFR pathway, which is not inhibited by these small kinase inhibitors.

Cetuximab is a monoclonal antibody that targets the extracelullar domain of the EGFR. In preclinical studies, the agent exerts antitumor and radiosensitizing effect in GBM. Furthermore, in intracranial GBM models, systemic administration of cetuximab has shown to be an effective treatment. ${ }^{6}$ Furthermore, cetuximab has shown high activity in tumors expressing EGFR and it is capable to induce VEGFR pathway inhibition. ${ }^{7,8}$ Indeed, preclinical data suggest that cetuximab binds to and internalizes EGFRvlll. This internalization induces an $80 \%$ reduction in active forms of EGFRvIII. ${ }^{9}$ Therefore, cetuximab therapy was a rational strategy for the treatment of tumors that express EGFR and EGFRvIII. At the present time, there is no available clinical data of cetuximab in patients with GBM.

A 47-year-old white male was diagnosed with GBM located at the right frontal lobe in September 2003 after an episode of seizure treated with phenytoin and steroids. The patient underwent complete surgical resection with residual microscopic positive margins. Surgery was followed by radiotherapy to a total of $45 \mathrm{~Gy}$ in four weeks and con- 
comitant treatment with temozolomide $\left(75 \mathrm{mg} / \mathrm{m}^{2}\right)$ for five consecutive days every 28 days. This was followed by temozolomide $\left(200 \mathrm{mg} / \mathrm{m}^{2}\right)$ for five consecutive days every 28 days until March 2004. In July 2004 a cranial MRI evidenced progressive disease according RECIST criteria and irinotecan $\left(250 \mathrm{mg} / \mathrm{m}^{2}\right.$ every 15 days $)$ was started. After two months of treatment, the patient condition deteriorated with worsening of a left hemiparesis and motor aphasia as well as radiological progression. The tumor was stained with EGFR antibodies targeted against extracellular EGFR domain and was found to be strongly positive with membranous and cytoplasmatic patterns. In September 2004, after informed consent was obtained, treatment with cetuximab, loading dose of $400 \mathrm{mg} / \mathrm{m}^{2}$, followed by a weekly dose of $250 \mathrm{mg} / \mathrm{m}^{2}$, was initiated.

After four weeks of treatment, patient symptoms began to improve (ECOG 4 to ECOG 1). A MRI scan performed eight weeks after treatment demonstrated a partial response in the tumor and considerable improvement in the surrounding edema (Fig. 1). Therefore, steroids were progressively tapered to a maintenance dose of $2 \mathrm{mg} /$ day of dexamethasone. The patient remained clinically and radiologically stable with weekly cetuximab until November 2005, when clinical and radiological worsening was detected (time to disease progression: 14 months).

Two additional patients, a 65-year-old male and a 42-year-old female, with primary EGFR positive GBM, located respectively at the right parietal lobe and the left occipital lobe, were consecutively treated. Both patients underwent maximal surgical resection (more than $90 \%$ tumoral mass), adjuvant temozolomide concomitant with radiotherapy and second line therapy with irinotecan. Then, progressive disease was diagnosed and they were treated with the same cetuximab schedule described above until progression. Clinical improvement and radiological stabilization was maintained for more than eight months (11 months and 13 months respectively). Only, phenytoin was administered as anticonvulsivant when needed.

The principal toxicities observed in these patients were, as expected, moderate asthenia and skin rash (grade 1-2 CTC-NCI v2.0), similar to those described in colorectal cancer studies. ${ }^{10}$ Although cross-reactivity with normal CNS antigens could be an inherent risk for cetuximab in this clinical setting, no new neurological symptoms, other than those related to the underlying disease, were observed in these patients.

We believe that the natural history of GBM in these three patients was clearly modified by cetuximab. Preclinical data demonstrating that EGFR blocking is an encouraging treatment strategy for patients with high-grade gliomas supports our results in this group of patients. Two potential mechanisms may be involved in this observation. First, cetuximab exerts a direct cytotoxic effect mediated by the blocking of EGFR in in vitro and in vivo studies. ${ }^{6}$ Second, blockage of EGFR by cetuximab induces an antiangiogenic effect inhibiting the vascular endothelial growth factor (VEGF) secretion as a consequence of a reduction of cellular level of hypoxia-inducible factor $1-\alpha .7,8$

Several factors limit the interpretation of these data. First, and most important, a clinical trial is required to confirm the responses observed in these three patients. The data, however, suggest a clear improvement in survival when compared with historical series. ${ }^{1,11}$
Second, the ability of a bulky monoclonal antibody to reach the CNS is not known. Several reports suggest that the blood brain barrier is presumably disrupted in patients with gadolinium-enhancing brain tumors. Although preclinical studies show that cetuximab crosses the blood brain barrier, it is unlikely that the cytotoxic effects of the antibody against the glioma cells are responsible for the entire clinical response observed. ${ }^{6}$ In this regard, other immunological mechanisms might be implicated. ${ }^{12}$ It is well described that secondary GBM have a better clinical course and could explain the survival observed in our patients. In this regard, all tumor samples were reevaluated by an expert neuro-pathologist and none of them presented the typical features of a secondary low-grade derived GBM. Other prognostic factors could be implicated in the evolution of our patients. All our patients were less than 60 years old at diagnosis. A retrospective analysis suggests a median survival of 43 weeks for this group. ${ }^{13}$ So, we consider that this prognosis factor has little influence in our results. Good performance status is correlated with improved survival in patients diagnosed with GBM. However, when cetuximab therapy was started, every patient has a bad performance status (ECOG 3-4) and the worst biological situation (second line was ineffective). Thus, it is questionable that performance status had any impact over our final data. Finally, the delay between the discontinuation of irinotecan and cetuximab initiation could be a confounding factor that limits our results. However, we consider that the rapid worsening (two months until progression) and the subsequent improvement of the clinical situation when cetuximab therapy was initiated do not support this hypothesis.

To the best of our knowledge, these are the first clinical and radiological responses reported for cetuximab in the treatment of human EGFR expressing GBM. In spite of these data, the effect of cetuximab remains indeterminate in the absence of a clinical trial. So, we have now initiated a phase II study evaluating the efficacy of this therapy in this patient population. 


\section{References}

1. Stupp R, Mason WP, van den Bent MJ, Weller M, Fisher B, Taphoorn MJ, Belanger K, Brandes AA, Marosi C, Bogdahn U, Curschmann J, Janzer RC, Ludwin SK, Gorlia T, Allgeier A, Lacombe D, Cairncross JG, Eisenhauer E, Mirimanoff RO, European Organisation for Research and Treatment of Cancer Brain Tumor and Radiotherapy Groups, National Cancer Institute of Canada Clinical Trials Group. Radiotherapy plus concomitant and adjuvant temozolomide for glioblastoma. N Engl J Med 2005; 352:987-96.

2. Leuraud P, Taillandier L, Medioni J, Aguirre-Cruz L, Criniere E, Marie Y, Kujas M, Golmard JL, Duprez A, Delattre JY, Sanson M, Poupon MF. Distinct responses of xenografted gliomas to different alkylating agents are related to histology and genetic alterations. Cancer Res 2004; 64:4648-53.

3. Rich JN, Hans C, Jones B, Iversen ES, McLendon RE, Rasheed BK, Dobra A, Dressman HK, Bigner DD, Nevins JR, West M. Gene expression profiling and genetic markers in glioblastoma survival. Cancer Res 2005; 65:4051-8.

4. Raizer JJ. HER1/EGFR tyrosine kinase inhibitors for the treatment of glioblastoma multiforme. J Neurooncol 2005; 74:77-86.

5. Mellinghoff IK, Wang MY, Vivanco I, Haas-Kogan DA, Zhu S, Dia EQ, Lu KV, Yoshimoto K, Huang JH, Chute DJ, Riggs BL, Horvath S, Liau LM, Cavenee WK, Rao PN, Beroukhim R, Peck TC, Lee JC, Sellers WR, Stokoe D, Prados M, Cloughesy TF Sawyers CL, Mischel PS. Molecular determinants of the response of glioblastomas to EGFR kinase inhibitors. N Engl J Med 2005; 353:2012-24.

6. Eller JL, Longo SL, Kyle MM, Bassano D, Hicklin DJ, Canute GW. Anti-epidermal growth factor receptor monoclonal antibody cetuximab augments radiation effects in glioblastoma multiforme in vitro and in vivo. Neurosurgery 2005; 56:155-62.

7. Luwor RB, Lu Y, Li X, Mendelsohn J, Fan Z. The antiepidermal growth factor receptor monoclonal antibody cetuximab/C225 reduces hypoxia-inducible factor-1 alpha, leading to transcriptional inhibition of vascular endothelial growth factor expression. Oncogene 2005; 24:4433-41.

8. Perera RM, Narita Y, Furnari FB, Gan HK, Murone C, Ahlkvist M, Luwor RB, Burgess AW, Stockert E, Jungbluth AA, Old LJ, Cavenee WK, Scott AM, Johns TG. Treatment of human tumor xenografts with monoclonal antibody 806 in combination with a prototypical epidermal growth factor receptor-specific antibody generates enhanced antitumor activity. Clin Cancer Res 2005; 11:6390-9.

9. Kang X, Patel D, Shi J, Hicklin D. Anti-EGFR monoclonal antibody cetuximab binds the EGFR variant III receptor and internalizes phosphorylated receptor on the cell surface. Eur J Cancer 2002; 38(Suppl 7), (Abstract 498).

10. Segaert $S$, Van Cutsem E. Clinical signs, pathophysiology and management of skin toxicity during therapy with epidermal growth factor receptor inhibitors. Ann Oncol 2005; $16: 1425-33$.

11. Grossman SA, Batara JF. Current management of glioblastoma multiforme. Semin Oncol 2004; 31:635-44.

12. Bleeker WK, Lammerts van Bueren JJ, van Ojik HH, Gerritsen AF, Pluyter M, Houtkamp M, Halk E, Goldstein J, Schuurman J, van Dijk MA, van de Winkel JG, Parren PW. Dual mode of action of a human anti-epidermal growth factor receptor monoclonal antibody for cancer therapy. J Immunol 2004; 173:4699-4707.

13. Grant R, Liang BC, Page MA, Crane DL, Greenberg HS, Junck L. Age influences chemotherapy response in astrocytomas. Neurology 1995; 45:929-33. 